\title{
Lipid peroxidation and antioxidant system in erythrocytes of brucella vaccinated and challenged
} \section{goats}

\begin{abstract}
The immune responses and protection in Brucella vaccination are mediated through interaction of Reactive oxygen species, free radicals and antioxidant system. These can be assessed by various oxidative stress and antioxidant biomarkers by measuring rate of lipid peroxidation (LPO) and activity of antioxidant enzymes in erythrocytes. In the present study interaction of Reactive oxygen species, free radicals and antioxidant system was evaluated during Rev.1 vaccination and challenge with virulent Brucella melitensis biovar 3 in adult healthy Brucella free pure bred Jamunapari goats. Vaccinated goats were challenged on $28^{\text {th }}$ day post vaccination. The level of Catalase, GST and MDA formation increased significantly whereas level of SOD and GSH reduced significantly after vaccination. The challenge with virulent culture on $28^{\text {th }}$ post vaccination initially reduced the blood erythrocyte level of Catalase, GST, GSH and MDA formation on $14^{\text {th }}$ day with subsequent increase on $28^{\text {th }}$ day post challenge excluding SOD and GSH. The erythrocyte level of catalase, GST and MDA formation reduced on $60^{\text {th }}$ day post vaccination. Based on mechanism involved in ROS production and antioxidant mechanism the levels of CAT and GST acted in synergism to immunization with Rev.1 by maintaining ROS in goat erythrocytes. As antioxidant system activation is required to obtain protection against Brucella, thus CAT and GST can be used as oxidative stress and antioxidant fingerprint markers of redox balance in brucellosis and its vaccination.
\end{abstract}

Keywords: 1, brucella melitensis, oxidative stress, antioxidant, biomarkers, erythrocytes, goat
Volume 4 Issue 5 - 2017

\author{
Amit Kumar,' Gupta VK, ${ }^{2}$ Verma AK, ${ }^{3}$ Rajesh \\ Mandil, ${ }^{4}$ Anu Rahal, ${ }^{5}$ Yadav SK' \\ 'Department of Veterinary Microbiology, DUVASU University, \\ India \\ ${ }_{2}^{2}$ Joint Director, Indian Veterinary Research Institute (IVRI), India \\ ${ }^{3}$ Department of Veterinary Epidemiology and Preventive \\ Medicine, DUVASU University, India \\ ${ }^{4}$ Department of Veterinary Pharmacology and Toxicology, \\ DUVASU University, India \\ ${ }^{5}$ Department of Goat Health, ICAR-Central Institute for \\ Research on Goats (CIRG), India
}

\section{Correspondence: Amit Kumar, Assistant Professor,} Department of Veterinary Microbiology, College of Veterinary Sciences, DUVASU, Mathura-28I00I, India, Tel +9194I2I 208 I3, Email balyan74@gmail.com

Received: July 07, 2017| Published: December 14, 2017
Abbreviations: ROS, reactive oxygen species; SOD, superoxide dismutase; CAT, catalase; GST, glutathione-s- transferase; GSH, glutathione; LPO, lipid peroxidation

\section{Introduction}

Brucellosis is a reemerging worldwide zoonotic disease and theoccurrence of the disease in humans is largely dependent on the animal reservoir. ${ }^{1}$ In human it is mainly caused by Brucella melitensis. The highest rate of human infection is mainly in those areas where rates of brucellosis in sheep and goats are high ${ }^{2,3}$ as sheep and goats are main reservoir of $B$. melitensis. ${ }^{4}$ The prevention and control of $B$. melitensis in sheep and goats can be the best fitted model to control human brucellosis. For the purpose it is important to understand the patho-physiological interface involved in the course of disease in sheep or goats as virulence of Brucella species varies according to species, strain and the number of brucellae in inoculums. ${ }^{5,6}$ Moreover, being intra cellular parasite, the pathogenic potential of Brucella spp. is highly dependent on its ability to enter and survive within host cells particularly polymorphonuclear cells and macrophages. ${ }^{7}$ Further for the survival ofBrucella spp. in the harsh environmental stress encountered in route of entry to their replicative niche, include interactive reactive oxygen species, acidic $\mathrm{pH}$, and nutrient deprivation as a key determinant of Brucella virulence. ${ }^{8,9}$ The capacity of Brucella to induce disease is dependent on their ability to overcome host cellular response and to replicate within both host phagocytes. ${ }^{10}$ In general the intracellular environment of any phagocytic cells is potentially hostile for microbes and, threat their viability by oxidative (Myeloperoxidase- $\mathrm{H} 2 \mathrm{O} 2$-halide) or nonoxidative (cationic protein, lysozyme, lactoferritin and proteases. ${ }^{11}$ Depending upon the type of adjuvants or immunomodulators used in any vaccine macrophages and dendritic cells are recruited and activated for antigen processing to generate free reactive radicals resulting in inflammatory sequelae. ${ }^{12}$ Superoxide dismutase (SOD), Catalase, and glutathione peroxidase are integral part of intracellular defense systems and antioxidant mechanism. ${ }^{13}$ Cytotoxic effects of oxidants involve DNA damage, protein oxidation, lipid peroxidation with inhibition of cellular metabolic pathways. ${ }^{12}$ The establishment of Brucella infection has been correlated with induced oxidative stress and lipid peroxidation in human, ${ }^{8}$ mice $;{ }^{11}$ cattle $;{ }^{14} \mathrm{rat}^{15}$ and in pregnant goats. ${ }^{16}$ Thus the aim of the present study was to assess erythrocyte redox parameters viz., lipid peroxidation, Glutathion (GSH) formation and activity of catalase, SOD and Glutathion-S- Transferase (GST) in clinically healthy goats during Rev.1 vaccination and challenge.

\section{Material and methods}

\section{Approval of IAEC}

The approval of study was obtained from Institutional Animal Ethics Committee (IAEC) of CIRG, Farha, Mathura (UP) and animals were maintained as per the guidelines of CPCSEA.

\section{Experimental Goats}

Pure bred apparently healthy non pregnant Brucella free Jamunapari adult female goats aged between 2-3years without any history of abortions were selected and screened twice at the interval of 28days for Brucella serum antibodies by RBPT; STAT; indirect ELISA and PCR based genus specific amplification from discharges and serum. The goats found negative were selected and separated for the study. 


\section{Vaccine and Challenge Strain}

Rev.1 vaccine consisting of Elberg 101 strain was obtained from Indian Immunological Limited (IIL), Hyderabad. Virulent bacterial strain Brucella melitensis biovars 3 (VTCCBAA228), submitted to NCBI, Data Bank and has been assigned the name of Brucella melitensis bv 3IND1was used to challenge vaccinated animals.

\section{Vaccination and challenge protocol}

The pure bred Jamunapari adult female goats $(n=3)$ were vaccinated with standard Rev.1 (IIL, Hyderabad) vaccine as per the manufacturer recommendations and challenged on $28^{\text {th }}$ day post vaccination with live virulent $B$. melitensis biovar $3 \mathrm{IND} 1$ cultures $\left(10^{9} \mathrm{CFU}\right)$ through subcutaneous route.

\section{Collection of blood samples}

The blood was collected on day of vaccination; $14^{\text {th }}, 28^{\text {th }}$ day of post vaccination and then $14^{\text {th }}, 28^{\text {th }}, 60^{\text {th }}$ and $90^{\text {th }}$ day post challenge.

\section{Estimation of parameters of oxidative stress in erythrocytes}

The RBC's separated from plasma by centrifugation were washed thrice with $0.15 \mathrm{M} \mathrm{NaCl}$. The RBC's suspension was divided in two parts. The first was used to prepare 33\% dilution of RBC's in PBS (pH 7.4). ${ }^{17}$ The washed erythrocyte pellets were suspended in PBS; $\mathrm{pH} 7.4\left\{\mathrm{NaCl}(8 \mathrm{~g}), \mathrm{KCl}(0.2 \mathrm{~g}), \mathrm{KH}_{2} \mathrm{PO}_{4}(0.2 \mathrm{~g})\right.$ and $\mathrm{Na}_{2} \mathrm{HPO}_{4}(0.94 \mathrm{~g})$ in about $800 \mathrm{ml}$ of distilled water and then volume was made to 1 litre with distilled water\} and kept at $4^{0} \mathrm{C}$ until further analysis. This $33 \%$ packed erythrocytes were used for the estimation of lipid peroxidation (LPO) and reduced glutathione (GSH). Then 1:10 dilution of packed erythrocytes in PBS ( $\mathrm{pH}$ 7.4) was prepared from the second part of RBC and was used for the estimation of Catalase (CAT) and Superoxide dismutase (SOD). Lipid peroxidation (LPO) and reduced gluthione (GSH) in cells were assayed on the day of blood collection. GSH was estimated by the 5, 5-dithiobis (2-nitrobenzoic acid) (DTNB) method. ${ }^{18}$ The extent of lipid peroxidation was evaluated in terms of malondialdehyde (MDA) production. ${ }^{19}$ Catalase was estimated in erythrocytes. ${ }^{20}$ The enzyme activities were also estimated for Superoxide dismutase (SOD) ${ }^{21}$ and Glutathione-S-transfer. ${ }^{22}$ To calculate the values of various enzyme activities the total protein content in erythrocytes were also measured. ${ }^{23}$

\section{Statistical analysis}

Various parameters were expressed as mean \pm SE. Mean of various parameters at different time intervals after vaccination and challenge were compared using ANOVA. ${ }^{24} \mathrm{~A}$ value of $\mathrm{P}<0.05$ was considered as statically significant.

\section{Results}

The goat erythrocytes were separated from blood samples for the estimation of level of LPO; GSH and activity of catalase, SOD and GST (Figure 1-5). The catalase level in erythrocytes increased after vaccination. Immediately after challenge it reduced significantly to reach 0day levels and then the highest level was observed on $28^{\text {th }}$ day post challenge with subsequent reduction in catalase activity (Figure1). Similar to catalase the activity of GST in goat erythrocytes also increased after vaccination with the highest activity on $28^{\text {th }}$ day post vaccination. The challenge with virulent pathogen initially reduced GST activity on $14^{\text {th }}$ day post challenge then it reached to its highest level on $28^{\text {th }}$ day post challenge with subsequent decline in activity. The GST activity was at the highest with significant difference on $28^{\text {th }}$ day post vaccination and challenge (Figure 2).
The SOD activity reduced significantly in all the animals up to $28^{\text {th }}$ day post vaccination and challenge. Thereafter it increased to reach nearly normal values (Figure 3 ). he lipid peroxidation level increased significantly in erythrocytes of goats in all the animals after vaccination. On challenge the LPO level was reduced initially and thereafter it increased significantly with the highest level on $28^{\text {th }}$ day post challenge. The MDA concentration further reduced to show low LPO level on $90^{\text {th }}$ day post (Figure 4). The reduction of glutathione was higher in vaccinated animals with the highest levels on day $14^{\text {th }}$ after vaccination. The challenge with virulent Brucella culture initially reduced GSH levels significantly. Then $14^{\text {th }}$ day post challenge onward the level of GSH recovered to normal levels (Figure 5).

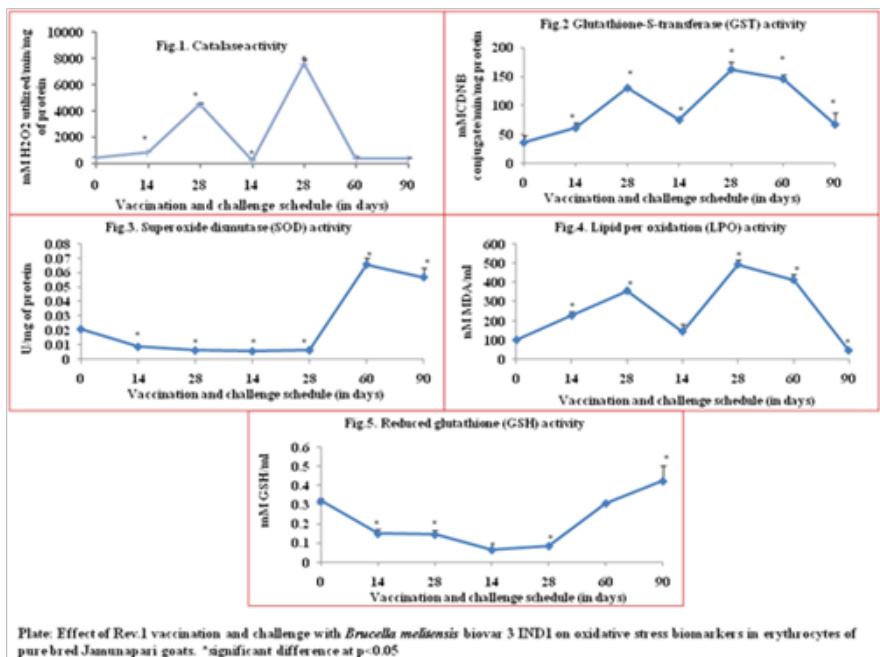

\section{Discussion}

Proper immune response require recruitment and activation of macrophages and dendritic cells for antigen processing and that leads to generation of free reactive radicals with ultimate inflammatory sequelae. ${ }^{6}$ Under normal physiological conditions, ROS are rapidly eliminated by antioxidant enzymes including catalase, superoxide dismutase (SOD), ${ }^{25,26}$ whereas GST play important role in the detoxification and/or excretion rate of biological compounds. ${ }^{27}$ In present study these changes were analyzed by monitoring oxidative stress and antioxidant biomarkers like lipid peroxidation (LPO), GSH formation and activity of antioxidant enzymes viz., Catalase, GST, SOD in erythrocytes of vaccinated animals. Catalase, SOD and GST are a part of intracellular defence systems against oxidation ${ }^{13}$ and cytotoxic effects of oxidants include protein oxidation and lipid peroxidation. ${ }^{12}$ In goat erythrocytes, CAT activity was increased (Figure 1) after vaccination and challenge in a similar pattern with peak values on $28^{\text {th }}$ day of post vaccination and challenge. These increases are expected due to activation of body immune system to remove the antigenic threat posed by vaccination and challenge by the production of toxic peroxides $\left(\mathrm{OH}, \mathrm{H}_{2} \mathrm{O}_{2}\right)$ and superoxide anion. ${ }^{12}$ These can be correlated with the increase concentration of MDA in erythrocytes (Figure 4) with highest values on $28^{\text {th }}$ day of post vaccination and challenge as CAT acts as preventive antioxidant and plays an important role in protection against the deleterious effects of lipid peroxidation. ${ }^{28}$ Further elevation of CAT is attributed to counteraction of scavenging of damaging toxic free radicals which may cause injury and damage. ${ }^{29}$ Increase in malondialdehyde levels are resulted from excessive production of free radicals during brucellosis and action of organism itself upon membrane lipids. ${ }^{14}$ Catalase and SOD are haemprotein and accomplish their antioxidant functions enzymatically by detoxifying the peroxides $\left(\mathrm{OH}, \mathrm{H}_{2} \mathrm{O}_{2}\right)$ and 
superoxide anion. ${ }^{26}$ Interestingly during the study the erythrocytic SOD levels reduced after vaccination and challenge and increased after $28^{\text {th }}$ day post challenge, a pattern reversed to CAT activity (Figure 3). Superoxide dismutase converts superoxide into $\mathrm{H}_{2} \mathrm{O}_{2}$ even then lower activity of SOD during vaccination and challenge reflects the lower production of superoxide anion during Brucella vaccination and challenge as reported earlier that Brucella does not modify free radical scavenging enzyme SOD activities. ${ }^{30}$ GST removes the excess free radicals from the system in the presence of GSH and GSH is an important first nonenzymic intracellular protective mechanism against various noxious stimuli including oxidative stress. ${ }^{31}$ The goat erythrocytes revealed reduction in GSH levels during vaccination and challenge with recovery after $60^{\text {th }}$ day post challenge (Figure $5)$. These lower levels of GSH in erythrocytes may be attributed to higher levels of GST (Figure 2) as an increased GST activity implies the consumption of GSH through the GST catalyzed reaction and play role in the detoxification of free radicals and electrophiles generated. ${ }^{6,32}$ Further such reduction of GSH levels are suggestive of impairment of cellular defense against ROS and may result in per oxidative injury. ${ }^{32}$ Thus the increasing pattern of CAT and GST are suggestive of removal of excessive level of free radicals from system and appear to work in the synergism of immune response required to overcome Brucella infection.

\section{Conclusion}

The present study revealed that free radicals produced during the Rev.1 vaccination are duly managed by antioxidant system and ROS produced during vaccination are well catalyzed by CAT and GST activity. CAT increases according to lipid peroxidation induced by live vaccine where as GST maintain the lower GSH level during vaccination. When vaccinated animals were challenged with live virulent Brucella, the oxidative stress induced by initial localization in liver in the form of increased MDA formation also revealed increase in CAT activity to overcome damage due to toxic substances like MDA. Similarly GST levels also revealed the synergistic activity of antioxidant system to overcome ROS and induce protection.

\section{Conflicts of interest}

There is no conflict of interest.

\section{Acknowledgements}

The authors are thankful to Dean and Vice Chancellor, DUVASU; Head, Division of Goat Health and Director, CIRG for the support and financial assistance provided to complete the study.

\section{Funding}

None.

\section{References}

1. Blasco JM. A review of the use of B. melitensis Rev. 1 vaccine in adult sheep and goats. Prev Vet Med. 1997;3(3-4)1:275-283.

2. England T, Kelly L, Jones R, et al. A simulation model of brucellosis spread in British cattle under several testing regimes. Prev Vet Med. 2004;63(1-2):63-73.

3. Pal M, Gizaw F, Fekadu G, et al. Public Health and Economic Importance of Bovine Brucellosis: An Overview. 2009;5(2):27-34.

4. Kumar A, Gupta VK, Verma AK, et al. Vaccines for caprine brucellosis: status and prospective. Int J Vaccines Vaccin. 2006;2(3):00030.
5. Kumar A, Gupta VK, Verma AK, et al. Assessment of hematological bio markers during vaccination and challenge of Brucella melitensis in goats. Int J Vaccines Vaccin. 2007;4(2):00078.

6. Rahal A, Kumar A, Singh V, et al. Oxidative stress, prooxidants and antioxidants: the Interplay. Bio Med Res Int. 2014;1-19.

7. Grillo MJ, Blasco JM, Gorvel JP, et al. What have we learned from brucellosis in the mouse model? Vet Res. 2012;43:29.

8. Köhler S, Michaux-Charachon S, Porte F, et al. What is the nature of the replicative niche of a stealthy bug named Brucella? Trends Microbiol. 2003;11(5):215-219.

9. Roop RM, Bellaire BH, Valderas MW, et al. Adaptation of the brucellae to their intracellular niche. Mol. Microbiol . 2004;52(3):621-630.

10. Kohler S, Foulongne V, Ouahrani-Bettache S, et al. The analysis of the intramacrophagic virulome of Brucella suis deciphers the environment encountered by the pathogen inside the macrophage host cell. Proc Natl Acad Sci USA. 2002;99:15711-15716.

11. Hornback ML, Roop RM. The Brucella abortus xthA-1 gene product participates in base excision repair and resistance to oxidative killing but is not required for wild-type virulence in the mouse model. $J$ Bacteriol. 2006;188(4):1295-1300.

12. Kim HS, Caswell CC, Foreman R, et al. The Brucella abortus general stress response system regulates chronic mammalian infection and is controlled by phosphorylation and proteolysis. J Biol Chem. 2013;288 (19):13906-13916.

13. Wagner BA, Buttner GR, Burns CP. Free radical mediated lipid peroxidation in cell oxidizability is a function of cell lipid bisallylic hydrogen content. Biochem. 1994;33(15):4449-4453.

14. Nisbet C, Yarim GF, Ciftci A, et al. Investigation of serum nitric oxide and malondialdehyde levels in cattle infected with Brucella abortus. Vet J of Ankara Univ. 2007;514(3):159-163.

15. Erdogan S, Aslantas O, Celik S, et al. The effects of increased cAMP content on inflammation, oxidative stress and PDE4 transcripts during Brucella melitensis infection. Res Vet Sci. 2007; 84(1):181-186.

16. Al Khafaji WS, Al-Farwachi MI. Antioxidant status in pregnant ewes vaccinated with Rev .1 against brucellosis. Iraqi Journal of Veterinary Sciences. 2012;26(1):15-19.

17. Yagi K. Lipid peroxides and human diseases. Chem Phys Lipids. 1978;45(2-4):337-351.

18. Prins HK, Loos JA. Glutathione. In: Yunis JG (Ed) Biochemical methods in red cell genetics. Academic Press, USA. 1969. P.127-129.

19. Rehman SU. Lead induced regional lipid peroxidation in brain. Toxicol Lett. 1984;21:333-337.

20. Bergmeyer HU. UV method of catalase assay. In: Methods of enzymetic analysis. Weinheim \& Decrfield Beah ( $\left.3^{\text {rd }}\right)$, USA. 1983. p.1-273.

21. Madesh M, Balasubramanian KA. Microtiter plate assay for superoxide dismutase using MTT reduction by superoxide. Indian $J$ Biochem Biophys. 1998;35:184-188.

22. Habig WH, Pabst MJ, Jakoby WB. Glutathione-S-transferases. The first enzymatic step in mercapturic acid formation. $J$ Biol Chem. 1974;249(22):7130-7139.

23. Lowry OH, Rosenbrough NJ, Farr AL, et al. Protein measurement with the folin phenol reagent. J Biol Chem. 1951;193(1):265-275.

24. Snedecor GW, Cochran WG. Statistical methods. ( $7^{\text {th }}$ edn), Oxford and IBH Publishing company, India. 1989.

25. Balasubashini MS, Rukkumani R, Viswanathan P, et al. Ferulic acid alleviates lipid peroxidation in diabetic rats. Phytother Res. 2004;18(4):310-314. 
26. Zhang X, Xie P, Li D, et al. Time-ependent oxidative stress responses of crucian carp (Carassius auratus) to intra peritoneal injection of extracted microcystins. Bull Environ Contam Toxicol. 2009;82(5):574-578.

27. $\mathrm{Lu}$ FC. Biotransformation of toxicants. In: Basic toxicology: fundamentals, target organs and risk assessment. ( $3^{\text {rd }}$ edn), Taylor $\&$ Francis, USA. 1996. p.27-39.

28. Dinkova-Kostova AT. Protection against cancer by plant phenyl propenoids: induction of mammalian anticarcinogenic enzymes. Mini Rev Med Chem. 2002;2:595-610.

29. Verma RS, Mnugya A, Srivastava N. In vivo chlorpyrifos induced oxidative stress: attenuation by antioxidant vitamins. Pest Biochem Physiol. 2007;88(2):191-196.
30. Melek IM, Erdogan S, Celik S, et al. Evaluation of oxidative stress and inflammation in long term Brucella melitensis infection. Molecular and Cellular Biochem. 2006;293(1-2):203-209.

31. Gross A, Bertholet S, Mauel J, et al. Impairment of Brucella growth in human macrophagic cells that produce nitric oxide. Microb Pathology. 2004;36(2):75-82.

32. Jackie T, Haleagrahara N, Chakravarthi S. Antioxidant effects of Etlingeraelatior flower extract against lead acetate induced perturbations in free radical scavenging enzymes and lipid peroxidation in rats. $B M C$ Research Notes. 2011;4:67. 